Erschienen in: Konopka, Marek/Strecker, Bruno (Hrsg.): Deutsche Grammatik

- Regeln, Normen, Sprachgebrauch. - Berlin, New York: de Gruyter, 2009. S.

70-87. (Institut für Deutsche Sprache. Jahrbuch 2008), https://doi.org/10.1515/9783110217360.2.70

\title{
Peter EisenberG*
}

\section{Schweigt stille, plaudert nicht}

Der öffentliche Diskurs über die deutsche Sprache ${ }^{1}$

Sehr geehrter Herr Oberbürgermeister, sehr geehrte Vertreter der Stadt Mannheim

und des Bibliographischen Instituts, verehrtes Preisgericht, lieber Herr Kollege Löffler, liebe Kolleginnen und Kollegen, Familie und Freunde, sehr verehrte Frau Lee und sehr geehrter Herr Meister, meine Damen und Herren,

vor allem anderen freue ich mich darüber, dass Sie anwesend sind, um die Verleihung des Konrad-Duden-Preises gemeinsam mit dem Geehrten zu feiern. Am liebsten würde ich die gesamte Redezeit dazu verwenden, jeden von Ihnen zu begrüßen, um dieser Freude Ausdruck zu geben. Zur Freude gehören auch die bewegenden Glückwünsche, geschrieben, gesprochen und in allen Registern dazwischen. Wir Wissenschaftler hören selten etwas darüber, wie unsere Bemühungen verstanden werden. Nun ist es gut damit. Nicht verschweigen will ich ein paar Vorhalte an meine Adresse, teilweise hart formuliert und ohne Absender expediert. Eisenberg könne den Preis nicht annehmen. Er trage Mitschuld daran, dass die gelungene Neuregelung der Orthografie von 1996 rückgebaut worden sei. Aber auch: Eisenberg trage Mitschuld daran, dass wir die alte Orthografie nicht ganz zurückbekommen haben. Ein bisschen irritiert war ich schon, einen Moment lang. Inzwischen freue ich mich nur noch. Schon, weil Sie da sind.

* Der Beitrag wird mit freundlicher Genehmigung des Dudenverlags in diesen Band aufgenommen. Die Erstpublikation der Preisrede erfolgte in Heft 59 der Reihe „DudenBeiträge zu Fragen der Rechtschreibung, Grammatik und des Stils", herausgegeben von der Dudenredaktion unter Leitung von Matthias Wermke.

1 Für den Druck leicht überarbeiteter Text der Preisrede vom 12.3.2008. Allen, die mit Rat und Auskünften geholfen haben, danke ich herzlich. 


\section{Causerie: Plaudern über die Sprache}

Über die deutsche Sprache wird viel und viel Beliebiges geredet. Man kann niemandem das Maul verbieten, aber es ist einen Versuch wert, die Sprache vor einigen ihrer Freunde in Schutz zu nehmen. Selbst wehrt sie sich ja kaum einmal.

Das Thema der Preisrede sollte eigentlich lauten „Reden ist Silber“. Meine Frau machte mich dann auf den Titel der Kantate BWV $211^{2}$ aufmerksam, und in der Tat passt plaudern noch besser als reden. Bach verwendet es ungefähr mit der Hauptbedeutung nach dem Grimm'schen Wörterbuch. ${ }^{3}$ Plaudern bedeutet

Gesprächig, traulich schwatzen, dann auch mit tadelndem nebensinne des albernen, ungehörigen schwatzens und klatschens oder des ausschwatzens von heimlichkeiten

zum Beispiel:

albern, wie ein stutzer plaudern (Die Räuber)

auf! oder ihr seid verloren,

unnützes zagen, zaudern und plaudern (Faust)

Das Geplauder hat etwas Bösartiges, Unablässiges und Leichtfertiges. Es trifft vieles, aber nicht alles genau, worüber ich sprechen möchte. Im fast unerschöpflichen Vorrat an Verba Dicendi haben wir beispielsweise noch sprechen, erzählen, bemerken, behaupten, bekunden, mitteilen, daherreden, verzapfen, sich verbreiten, fabulieren, plauschen, plappern, quatschen, klatschen, quasseln, faseln, schwatzen, schwätzen, schwafeln, schwadronieren, brabbeln, ratschen, tratschen und so weiter. ${ }^{4}$ Versuchen Sie einmal, das passende Verb zu finden, wenn Sie im öffentlichen Diskurs über das Deutsche Folgendes hören oder lesen.

„Sie müssen sich doch nur ansehen, wer dieses Geschwafel in unserem Land eigentlich verzapft. ... Das sind Leute, die glauben, ihre Halbbildung könnten sie dadurch steigern, daß sie sich ... plötzlich nicht mehr in Deutsch ausdrücken." 5

Das Jahr der Geisteswissenschaften (2007) sollte ,sich ganz besonders der Sprache widmen." Denn die Spezialisierung der Fächer bringe je eigene Sprachen hervor mit der Folge, ,dass die Sprache fehlt, Sprachlosigkeit ausgebrochen ist oder aber ein Sprachenwirrwarr herrscht." 6

2 Schmieder, Wolfgang: Thematisch-systematisches Verzeichnis der Werke Johann Sebastian Bachs. Leipzig 1961 (3. Auf1.), S. 283.

3 Deutsches Wörterbuch von Jacob und Wilhelm Grimm. Siebenter Band. Leipzig 1889. Nachdruck München 1984, Band 13, S. 1928 f.

4 Dornseiff, Franz: Der deutsche Wortschatz in Sachgruppen. Berlin 2004 (8. Auf1.), S. 228.

5 Helmut Schäfer (FDP), von 1987 bis 1998 Staatsminister im Auswärtigen Amt. Zitat aus dem Netz.

6 Bundesministerin Schavan bei der Eröffnung des Leibniz-Tages der Berlin-Brandenburgischen Akademie der Wissenschaften am 5. Mai 2006. Im gesamten Jahr der Geisteswissenschaften hat m.W. niemand auch nur eine Andeutung darüber gemacht, was mit Sprachlosigkeit oder Sprachenwirrwarr gemeint sein könnte. 
Im Mittelpunkt des Schulwettbewerbs zum Jahr der Geisteswissenschaften steht „die Frage nach Sprache als Fundament der Welterschließung und als unverzichtbare Basis von Denken und Mitteilen.“7

„Hubert Mania hat das Glück gehabt, von einer Zeit zu handeln, als in Deutschland nicht nur den großen Autoren eine bedeutende Sprache zur Verfügung stand." 8

„Wenn wir alle anfangen, Englisch zu singen, verleugnen wir unsere Wurzeln." "Nur deutsche Musik geht dem Menschen an die Seele." 10

Im öffentlichen Sprachdiskurs greift man verbal bedenkenlos in eine der oberen Schubladen. Starke Worte kosten nichts, sie verpflichten zu wenig, sie begleiten uns auf Schritt und Tritt. Ihre Wirkung beruht vor allem auf dem Appell an je spezifische Ressentiments.

Öffentliches Geplauder findet sich selten dort, wo wissenschaftliche Disziplinen ihren Gegenstand für sich haben, auch wenn an ihm ein öffentliches Interesse besteht. Das Interesse an einigen Informations- oder Naturwissenschaften, aber auch einigen Geisteswissenschaften besteht in erster Linie an den Ergebnissen und natürlich den Kosten, die mit Forschung verbunden sind. Was den Gegenstand betrifft, bleibt die Wissenschaft jedoch weitgehend autonom.

Die Sprachwissenschaft gehört nicht zu diesen Disziplinen. Der Diskurs richtet sich auf ihren Gegenstand, eben die Sprache, viel weniger auf Ergebnisse der Sprachwissenschaft. Unser Gegenstand gehört uns nicht allein. Das birgt Chancen wie Risiken für unsere Disziplin, die meiner Meinung nach nur zum Teil ausreichend reflektiert sind. Die Verleihung des Konrad-DudenPreises ist ganz bestimmt ein guter Anlass, über diese Frage nachzudenken, steht das Werk von Konrad Duden doch ebenso wie der Duden mit je einem Bein in der Wissenschaft und in der Öffentlichkeit. ${ }^{11}$

Das umso mehr, als es nicht um die Sprache allein, sondern oft genug auch um die Sprachwissenschaft geht. Hört man uns nicht, heißt es etwa: „Die Wis-

7 Mal haben wir gar keine Sprache (Fn. 6), dann ist sie wieder das Fundament der Welterschließung. Wenn beide Einsichten wie hier aus derselben Quelle fließen, bleibt nur Geplauder.

8 Burkhard Müller zur Biografie von Hubert Mania über Karl Friedrich Gauß. In: Süddeutsche Zeitung vom 1./2. März 2008, S. 17. Was meint der Rezensent wohl mit der Feststellung, in Deutschland habe man zu Lebzeiten von Gauß das Glück einer bedeutenden Sprache gehabt? Wie steht es mit der Sprache, die dem Rezensenten heute zur Verfügung steht?

9 Udo Jürgens, immer wieder einmal.

10 Ute Freudenberg, immer wieder einmal.

11 Der „Wahrig“ teilt selbstverständlich dieses Schicksal. Für einen Wissenschaftler aus unserem Fach gibt es wenig bessere Möglichkeiten, seine Auffassungen in den öffentlichen Diskurs einzubringen, als durch die Kooperation mit Duden und Wahrig. Der Vorwurf, es sei schon schlimm, mit dem Duden zu kooperieren, und noch schlimmer sei es - wie ich es seit Jahren tue - mit Duden und Wahrig zu kooperieren, bleibt mir unverständlich. 
senschaft, die es wissen sollte, hält sich bedeckt. Die akademische Linguistik scheint schon die Vorstellung, ein Sprachgebrauch könnte besser sein als der andere, albern zu finden. “12 Oder etwas härter: „Die Sprachwissenschaft und die sprachpflegenden Institutionen haben ... sich ... aus ihrer Verantwortung für unsere Sprache gestohlen." 13

Sagen wir etwas Unüberhörbares, wird man noch direkter: „Nur haben Konrad Dudens sel. Nachfolger mit ihren Versuchen, die Sprache zu betonieren, noch nie viel Glück gehabt, und dabei soll es auch bleiben" 14 , oder auch so: „Wie wäre es, wenn die Linguisten sich deshalb ganz aus den sprachpflegerischen Bemühungen der Bürger heraushielten und diese gewähren ließen? Die kommen bisher gut ohne linguistische Beratung aus ..." ${ }^{15}$ Das könnte Euch so passen.

Noch ein Letztes vorweg. Fast jeder Gegenstand lässt sich so darstellen, dass er nach einem halb vollen oder aber nach einem halb leeren Glas aussieht. Bei Sprachen wie dem Deutschen ist es sogar ohne Weiteres möglich, das Glas fast ganz voll oder fast ganz leer erscheinen zu lassen. In den nächsten sieben Minuten möchte ich versuchen, das Glas ein wenig zu füllen, ohne etwas einfach Unzutreffendes zu sagen. Es geht einmal um Fakten, die das Deutsche und seine Verwendung betreffen, zum anderen darum, wie viel man über diese Sprache weiß. Wie sieht der Gegenstand aus, über den so gern geplaudert wird?

\section{Eine große, weit ausgebaute und vielgestaltige Sprache}

\subsection{Große Zahl von Sprechern und Lernern}

Mit etwas weniger als hundert Millionen Sprechern liegt das Deutsche weit hinter Sprachen wie Chinesisch, Spanisch oder Englisch. ${ }^{16}$ Ein anderes Bild ergibt sich aber, wenn man die Gesamtsituation auf der Erde betrachtet. Man rechnet heute noch mit etwa 6000 gesprochenen Sprachen. Nach den Kriterien der UNESCO gelten weniger als 400 von ihnen als nicht gefährdet. Diese Sprachen haben mehr als eine Million Sprecher und werden insgesamt von ungefähr 95\% der Weltbevölkerung gesprochen. Gemessen an der Zahl der Sprecher ist das Deutsche mit Abstand die größte Sprache der Europäischen

12 Zimmer, Dieter E.: Alles eine Sache des Geschmacks? Von wegen! In: DIE ZEIT Nr. 31 vom 26. Juli 2007, S. 43.

13 Glück, Helmut/Krämer, Walter (Hg.): Die Zukunft der deutschen Sprache. Eine Streitschrift. Leipzig 2000, S. 90.

14 Thalmayr, Andreas (d. i. Enzensberger, Hans Magnus): Heraus mit der Sprache. München 2005, S. $11 \mathrm{f}$.

15 Schrammen, Gerd: Gelehrte Anglizismenblindheit. In: Sprachnachrichten Nr. 36/Dezember 2007, S. 22.

16 Viele Zahlen in: Ammon, Ulrich: Die internationale Stellung der deutschen Sprache. Berlin 1991. Das Werk soll demnächst in aktualisierter Fassung vorliegen. 
Union und befindet sich in der Gruppe des ersten Dutzends oder der ersten zwei Promille (nicht Prozent!) der Sprachen der Erde.

Das Deutsche wird weltweit von ungefähr 17 Millionen Nichtmuttersprachlern gelernt. Damit liegt es ebenfalls weit hinter dem Englischen, dem Spanischen und jetzt auch dem Chinesischen. Die Situation ist in den Weltgegenden unterschiedlich. In vielen Regionen konkurriert das Deutsche als zweite Fremdsprache mit anderen westeuropäischen Sprachen, aber auch mit dem Chinesischen, Japanischen und Arabischen. Das Deutsche gehört zu den wenigen Sprachen, die bis heute weltweit gelernt werden. Innerhalb der Europäischen Union belegt es als Lernersprache den zweiten Platz nach dem Englischen und vor dem Französischen.

\subsection{Weit fortgeschrittener Ausbau}

Wie weit eine Sprache ausgebaut ist und sich insofern zu anderen Sprachen ins Verhältnis setzen lässt, ist keineswegs offensichtlich. Man kann versuchen, den Stand des Ausbaus an einfachen Kriterien wie Größe des Wortschatzes oder Differenziertheit von Morphologie und Syntax festzumachen. Aber auch das ist schwierig. Was den Wortschatz betrifft, werden ja immer wieder abenteuerliche Konkurrenzzahlen etwa über das Deutsche im Vergleich zum Englischen gehandelt. Es soll der Hinweis genügen, dass es aus guten Gründen keine verlässlichen Zahlen gibt, das Deutsche aber jedenfalls eine in dieser Hinsicht sehr wichtige Eigenschaft hat: Seine Morphologie ist - vielleicht mit Ausnahme der Inkorporations- und Univerbierungsprozesse - im Allgemeinen klar von der Syntax getrennt. Man weiß also ganz gut, wann etwas als Wort und wann etwas als Phrase zu gelten hat. Auf diesem Hintergrund lässt sich feststellen, das Deutsche verfüge über ein höchst produktives und vielfältiges Wortbildungssystem. Sein Reservoir an Wörtern ist praktisch unerschöpflich, auch wenn es nicht um Verba Dicendi geht. Bitte erinnern Sie sich daran, wie Herr Kollege Grosse dies in seiner Dudenpreis-Rede demonstriert hat. Auf die Frage, was er werden wolle, sagte sein Enkel „Ich werde Müllwagenhintendraufsteher." Da kann einem schwindelig werden. Oder nehmen Sie als beliebig herausgegriffenes Beispiel den Umfang des Goethe-Wörterbuchs. Man war lange Zeit der Auffassung, Goethes Wortschatz liege bei etwa 40000 Wörtern. Heute, bei fortgeschrittener Arbeit, geht man von 90000 Wörtern aus. ${ }^{17}$ Auch bei Berücksichtigung der notwendigen lexikologischen Relativierungen ${ }^{18}$ bleibt unbestreitbar: Das Deutsche hatte zu keiner Zeit einen größeren Wortschatz, als es ihn heute hat.

17 bibliothek.bbaw.delgoethe/woerterbuch

18 Harm, Volker: Gibt es eine Monosemierungstendenz in der Wortgeschichte des Neuhochdeutschen? Überlegungen zur sprachhistorischen Interpretation lexikographischer Befunde. In: Zeitschrift für germanistische Linguistik 29 (2001), S. 364-380. 
Noch schwieriger wird es beim eigentlichen Kern der Grammatik, der Morphosyntax. Niemand regt sich auf, wenn er etwa bei Peter von Polenz liest, ${ }^{19}$ die Syntax des Deutschen habe sich in den vergangenen zweihundert Jahren, also nach der Klassik, in einigen Bereichen weiter ausgefaltet. Es gibt auch Verluste, etwa beim verbregierten Genitiv oder bei der Kasusmarkierung substantivischer Nominalgruppen, aber insgesamt kann von einer Verarmung der Syntax keine Rede sein. Das Gegenteil ist richtig. Erst neuerdings wird versucht, dies genauer zu beschreiben und zu verstehen, etwa in Arbeiten von Cathrine Fabricius-Hansen, die das Deutsche aufgrund seiner syntaktischen Möglichkeiten zur Textgestaltung als reife Sprache bezeichnet hat und damit auch die Tür zu einer Diskussion öffnet, die seit einiger Zeit im Rahmen der Sprachtypologie über die Begriffe einfache vs. komplexe Sprache geführt wird. Diese heikle Diskussion qualifiziert das Deutsche keineswegs als in jeder Hinsicht besonders komplex, ihre vorläufigen Ergebnisse lassen aber die nichtimpressionistische Feststellung zu, dass die Syntax des Gegenwartsdeutschen mehr Möglichkeiten hat als in den früheren Sprachstufen und im Sprachenvergleich zu den sehr weit ausgebauten gehört. ${ }^{20}$

\subsection{Vielfältig verwendbar}

Die Vielfalt ihrer Verwendung sagt eine Menge über den Zustand einer Sprache, entsprechend facettenreich ist das Thema. Ich möchte nur zwei oder drei Verwendungsmerkmale nennen, die für den öffentlichen Diskurs von besonderer Bedeutung sind. Zunächst wieder ein Blick auf die globale Situation.

In ungefähr 2400 der 6000 Sprachen der Erde wurde die Bibel übersetzt, d.h. in mindestens dieser Zahl von Sprachen kann die Bibel auf die eine oder andere Weise niedergeschrieben werden. Für etwa 800 Sprachen gibt es so etwas wie einen Buchmarkt, ungefähr 300 von ihnen werden systematisch unterrichtet. Etwas mehr als 100 Sprachen besitzen den Status einer Staatssprache, aber nur gut die Hälfte davon verfügt über eine allgemein verwendbare Software. $^{21}$

Vielleicht doch überraschend gering ist die Zahl der Sprachen, die nach dem üblichen Verständnis keine Verwendungsbeschränkungen aufweisen. Weniger als zwei Dutzend verfügen über einen voll ausgebauten Varietätenraum mit Dialekten, Soziolekten, Wissenschafts- und Fachsprachen, Stan-

19 Polenz, Peter von: Deutsche Sprachgeschichte vom Spätmittelalter bis zur Gegenwart. Band III. 19. und 20. Jahrhundert. Berlin 1999, S. $338 \mathrm{ff}$.

20 Fabricius-Hansen, Cathrine: Deutsch - eine rreife Sprache. Ein Plädoyer für die Komplexität. In: Stickel, Gerhard (Hg.): Deutsch von außen. Berlin 2003, S. 99-112. Dahl, Östen: The Growth and Maintenance of Linguistic Complexity. Amsterdam/Philadelphia 2004.

21 Solche Zahlen finden sich beispielsweise im Beitrag von Mart Rannut (Tallin) zur Jahrestagung 2007 der European Federation of National Institutions for Language (EFNIL), die vom 12. bis 14.11.2007 in Riga stattfand. Die Kongressakten sind unter der Herausgeberschaft von Gerhard Stickel im Druck. 
dard-, Literatur- und Umgangssprache. Was die Variation betrifft, ist Deutsch „wahrscheinlich die vielgestaltigste Sprache Europas“22, was einerseits bemerkenswert, andererseits aber nicht erstaunlich ist, wenn man ihre Größe und geografische Platzierung in Rechnung stellt. Auf den Domänenverlust des Deutschen als Wissenschaftssprache komme ich etwas später zu sprechen. Im Augenblick soll es um den Stand der Literalisierung im deutschen Sprachgebiet gehen.

Zuerst zur Presse. Zustand und Verbreitung der Presse sind aus verschiedenen Gründen ein wichtiger Indikator, beispielsweise weil man häufig annimmt, die Presse setze das Maß für Standardisierung. Das muss und kann man genauer begründen, besonders dann, wenn gleichzeitig die traditionell verbreitete Auffassung fällt, das Maß werde von der schönen Literatur gesetzt. Lassen wir die Frage im Augenblick so stehen. Jedenfalls bilden die ungefähr 380 Tages- und Wochenzeitungen in Deutschland mit einer Gesamtauflage von über 26 Millionen Exemplaren eine der dichtesten und differenziertesten Presselandschaften der Erde. Damit befinden wir uns ungefähr auf dem Niveau von 1989. In den ersten Jahren nach der Wende lagen alle Zahlen höher. ${ }^{23}$

Dann das seit langem totgesagte Buch. In deutscher Sprache erscheinen jährlich ungefähr 100000 Buchtitel, deren Vielfalt die des Varietätenraums spiegelt und insbesondere die Varietät Literatursprache einschließt. ${ }^{24} \mathrm{Es}$ ist eine Aufgabe für sich, auch nur im Ansatz zu verdeutlichen, was dies für die deutsche Sprache bedeutet. Immerhin erweisen auch die größten Sprachpessimisten der Literatursprache ihre Referenz, manchmal sogar mit einem Zungenschlag wie „Zum Glück haben wir ja noch die Dichter und Schriftsteller." 25

Für das Sprachliche im engeren Sinn ist von gleicher Bedeutung das Übersetzen. Nach Erhebungen des Börsenvereins des deutschen Buchhandels wird in keine Sprache der Erde so viel übersetzt wie ins Deutsche. ${ }^{26}$ Wer sich einmal in dieser Szene umtut, kann von der Intensität, Härte und Vielfalt der

22 Berend, Nina: „Deutsch ist wahrscheinlich die vielgestaltigste Sprache Europas ...". Zum Projekt `Standardvariation « im IDS. In: Sprachreport 22 (2006), H. 4, $10-12$.

23 Die deutschen Zeitungen in Zahlen und Daten. Auszug aus dem Jahrbuch ,Zeitungen 2007“. Herausgeber: Bundesverband Deutscher Zeitungsverleger. Markgrafenstr. 15, 10969 Berlin. (www.bdzv.delbrochuere.html). S.a. World Association of Newspapers. World Press Trends 2007 (www.wan-press.org/article15362.html).

24 www.boersenverein.deldel 64586

25 So z. B. Jürgen Trabant: Die gebellte Sprache: Über das Deutsche. In: Berlin-Brandenburgische Akademie der Wissenschaften. Berichte und Abhandlungen, Band 13. Berlin 2007, S. 315. Ähnlich, aber ohne Verweis vonseiten des Autors, auch: Trabant, Jürgen: Die gebellte Sprache. Aufgeputzt und abgewirtschaftet: Der deutsche Sonderweg von der Scham der frühen Jahre zur postnationalen Sprachgemeinschaft. In: Frankfurter Allgemeine Zeitung vom 28.9.2007, S. 40.

26 Auch im Index Translationum der UNESCO rangiert das Deutsche als Zielsprache mit einigem Abstand vor dem Spanischen, Französischen, Englischen und Japanischen. 
geleisteten Spracharbeit nur beeindruckt sein. Beim Übersetzen wird ja auch ausgelotet, wie weit man Finessen anderer Sprachen und der in ihnen möglichen Stile mit den Mitteln des Deutschen einfangen kann.

\section{Eine der am besten beschriebenen Sprachen}

Lieben wir unsere Sprache? Kein Sprachwissenschaftler wird so fragen. Jedoch: Wie weit sich eine Sprachgemeinschaft auf ihre Sprache einlässt, wird sicher auch daran deutlich, wie viel sie über ihre Sprache weiß und wie viel von diesem Wissen allgemein verfügbar ist. Ein solches Wissen - oder was dafür gehalten wird - bestimmt den öffentlichen Sprachdiskurs in unterschiedlicher Form mit Sprachratgebern, Glossen und essayistischen Darstellungen jeder Art. Sprachwissenschaftlich geht es in erster Linie um Grammatiken und Wörterbücher. ${ }^{27}$

Wie viele Sprachen grammatisch gut beschrieben sind, ist unerwartet schwer zu ermitteln. ${ }^{28}$ Man schätzt, dass es für weniger als fünf Prozent zwei oder drei Grammatiken von mittlerem Umfang gibt. Natürlich ist grammatisches Wissen nicht nur in solchen Büchern niedergelegt. Im größten Sprachatlas überhaupt sind für durchschnittlich je 400 Sprachen jeweils 142 grammatische Merkmale in einem aufwendigen Verfahren und teilweise unabhängig von vorliegenden Grammatiken zusammengetragen worden. ${ }^{29} \mathrm{Ähn}-$ liches lässt sich von den Projekten zur Dokumentation bedrohter Sprachen sagen. ${ }^{30}$ Grammatiken in Buchform spielen aber nach wie vor ihre besondere Rolle, was das Wissen über eine Sprache betrifft. Sie sind in dieser Hinsicht allenfalls vergleichbar mit bestimmten Wörterbüchern.

Für das Deutsche liegt, beginnend mit dem Jahr 1965, eine ausführliche Dokumentation zur wissenschaftlichen Grammatik vor, und zwar als Buch

27 Der Wille zum Wissen über eine Sprache kann sicher als ein spezieller Aspekt von Sprachloyalität angesehen werden, obwohl man diesen im Allgemeinen nicht in Rechnung stellt, vgl. z. B. Klein, Wolf Peter: Fehlende deutsche Sprachloyalität? Tatsachen und Überlegungen zur jüngsten Entwicklung des öffentlichen Sprachbewusstseins in Deutschland. In: Linguistik online 9 (2001/2).

28 Die umfangreichste Bibliografie wird von Harald Hammarström geführt (www.cs. chalmers. se/ harald2). Der Kölner Typologe Hans-Jürgen Sasse schätzt, dass etwa die Hälfte der heute gesprochenen Sprachen „anständig dokumentiert" ist (Süddeutsche Zeitung vom 4.2.2008, S.12). Der Leipziger Typologe Martin Haspelmath schreibt (Elektropost vom 26.1.2008): ,So gut wie die großen europäischen Nationalsprachen sind nur noch das Japanische, Chinesische und Arabische beschrieben; schon das Hindi fällt deutlich zurück." Am Leipziger Max-Planck-Institut für evolutionäre Anthropologie macht man sich Gedanken über ein Projekt zur Dokumentation der bekannten Fakten.

29 Haspelmath, Martin/Dryer, Matthew/Gil, David/Comrie, Bernard (Hg.): The World Atlas of Language Structure. Oxford 2005.

30 Es gibt inzwischen mehrere große Projekte, die an der Dokumentation bedrohter Sprachen arbeiten. Informationen z.B. über die Gesellschaft für bedrohte Sprachen, www.uni-koeln.delgbs/ 
wie in elektronischer Form. ${ }^{31}$ Die Bibliografie weist über 500 Arbeiten jährlich aus. Für die Zeit zwischen 1965 und 2002 finden sich mehr als 50 umfangreiche Übersichtswerke und Gesamtdarstellungen, darunter mehrere von nichtdeutschen Autoren, mehrere kontrastive und mehrere mit spezieller Perspektive, beispielsweise als rezeptive Grammatik, didaktische Grammatik oder Textgrammatik. Man geht kein Risiko mit der Feststellung ein, das Deutsche gehöre zu den grammatisch am besten beschriebenen Sprachen überhaupt. Und die Diskussion darüber, welche Grammatiken man brauche, ist keineswegs abgeschlossen. Gespannt warten wir darauf, wann die Forderung nach einer Grammatik der gesprochenen Sprache Wirklichkeit wird und wie sich die Bemühungen um eine integrierte Grammatik von Laut- und Gestensprache entwickeln werden. ${ }^{32}$

Ähnliche Schwierigkeiten wie bei der Grammatikrecherche hat es bei den Wörterbüchern gegeben. Es ist mir nicht gelungen, eine griffige Übersicht zu gewinnen. Wohl die Hälfte der Bibelsprachen habe ein Wörterbuch mit wenigstens einigen Tausend Einträgen: Dies ist noch die präziseste Auskunft, $\mathrm{zu}$ der sich erfahrene Lexikologen bewegen lassen. Wie viele Sprachen gelten als lexikografisch gut beschreiben? Der erste Mann am Platze schreibt: „Leider kann ich Ihre Frage nicht beantworten. Das kann wahrscheinlich niemand ... Man müsste zunächst wissen, was man unter einer lexikografisch wohlversorgten Sprache verstehen könnte. Dazu eine Definition zu basteln, wäre reizvoll." ${ }^{33}$ Ebenso aussichtslos ist es aber, die Zahl der Wörterbücher des Deutschen zu nennen. Sieht man sich nur die Systematik der lexikografischen Beschreibung des Deutschen in HSK $5^{34}$ oder in den drei vorliegenden Bänden der Bibliografie zu seiner Lexikografie ${ }^{35}$ an, dann bleiben auch für einen allgemeineren Bedarf wenig Wünsche offen. Wie andere gut beschriebene Sprachen hat das Deutsche seine lexikografischen Eigenheiten, sogar im Bereich der weitverbreiteten Wörterbücher. Seit Campe verfügt es über spezielle Fremdwörterbücher (was ja durchaus ambivalente Bewertungen mit sich bringt) und es verfügt wie nur wenige Sprachen über umfangreiche Wörterbücher zu den sprachlichen Zweifelsfällen. Im Erscheinen befindet sich das

31 Eisenberg, Peter/Gusovius, Alexander: Bibliographie zur deutschen Grammatik 1965 1983. Tübingen 1985. Eisenberg, Peter/Wiese, Bernd: Bibliographie zur deutschen Grammatik 1984-1994. Tübingen 1995. Frosch, Helmut/Schneider, Roman/Strecker, Bruno/ Eisenberg, Peter: Bibliographie zur deutschen Grammatik 1994-2002. Tübingen 2004. Frosch, Helmut/Schneider, Roman/Strecker, Bruno: Bibliographie zur deutschen Grammatik 2003-2007. Tübingen 2008. Die elektronische Fassung unter hypermedia.idsmannheim.de.

32 Fricke, Ellen: Grundlagen einer multimodalen Grammatik des Deutschen: Syntaktische Strukturen und Funktionen. Habilschrift Universität Frankfurt/O. 2008.

33 Herbert Ernst Wiegand in einer Elektropost vom 22.12.2007.

34 Hausmann, Franz Joseph/Reichmann, Oskar/Wiegand, Herbert Ernst/Zgusta, Ladislav (Hg.): Wörterbücher. Zweiter Teilband. Berlin/New York 1990, S. 2037-2246.

35 Herbert Ernst Wiegand: Internationale Bibliographie zur germanistischen Lexikographie und Wörterbuchforschung. Berlin. Bd. 1 und 2 2006, Bd. 3 2007, Bd. 4 im Druck. 
singuläre Wörterbuch des sprachlichen Gegensinns, ${ }^{36}$ ein digitales Großwörterbuch ${ }^{37}$ ist im Entstehen. Noch einmal: Niemand weiß, wie viele Wörterbücher des Deutschen es gibt.

So viel zur Beschreibung unserer Sprache. Falls der Eindruck entsteht, hier kapriziere sich jemand doch ein wenig einseitig auf an sich dürre Fakten, Zahlen und Vergleiche, dann ist er zu Recht entstanden. Denn man kann über eine Sprache eben so oder so reden. Was über das Deutsche geredet und geplaudert wird, sollte doch mit den dürren Fakten vereinbar bleiben. Deshalb werden sie herausgestellt. Bestimmte Einlassungen im öffentlichen Diskurs, dem wir uns jetzt zuwenden, können dann hoffentlich nicht einfach stehen bleiben.

Von den Themen des Sprachdiskurses greife ich einige der öffentlichsten heraus. In Kürze wird dargelegt und begründet, was ich als Sprachwissenschaftler gern in den Diskursen über Anglizismen, über Deutsch als Wissenschaftssprache und schließlich über die angebliche Sprachscham der Deutschen zur Geltung bringen möchte.

\section{Fremdwörter: Sprache mit Kraft zur Integration}

Für die These vom Sprachverfall durch schlechten Sprachgebrauch spielen die Anglizismen nach wie vor die prominenteste Rolle. Immer wieder packen den normalen Sprachteilhaber Zorn und Ekel, wenn er sich der Sprachmacht ignoranter, prätentiöser, eitler und bewusst irreführender Lancierung von Anglizismen gegenübersieht, sei er nun Sprachwissenschaftler oder nicht. Einem Diskurs, der sich dieser Sprachmacht entgegenstellt, wünsche ich allen Erfolg. Allerdings hat der Homo politicus Vorbehalte gegenüber bestimmten Ausprägungen der Debatte, etwa wenn nicht präsent bleibt, dass man Auswirkungen der Globalisierung nicht am sprachlichen Symptom kurieren kann..$^{38}$ Erweckt jemand diesen Eindruck, handelt es sich um versuchten politischen Betrug.

Die besondere Aufgabe und Verantwortung der Sprachwissenschaft besteht darin, Auswirkungen des kritisierten Sprachgebrauchs auf die Sprache zu untersuchen und der Sprachgemeinschaft deutlich zu machen, was vor sich geht oder absehbar vor sich gehen wird. Dies zu wissen, ist Voraussetzung für jede Art von Sprachbewertung.

Die Sprachwissenschaft wird dieser Aufgabe gerecht. Wir verfügen über eine Reihe von Studien über das Verhalten von Anglizismen im Deutschen,

36 Rolf Peter Lutzeier: Wörterbuch des Gegensinns im Deutschen. Band 1, A-G. Berlin 2007.

37 Digitales Wörterbuch der deutschen Sprache bei der Berlin-Brandenburgischen Akademie der Wissenschaften: www. dwds. de.

38 In aller Deutlichkeit so z. B.: Stellungnahme zur Debatte über den zunehmenden Einfluß des Englischen auf die deutsche Sprache. In: Deutsche Akademie für Sprache und Dichtung. Jahrbuch 2001. Göttingen 2002, S. 9-16. 
deren Ergebnisse man so zusammenfassen kann: ${ }^{39}$ Die grammatische Integration von Anglizismen verläuft im Allgemeinen problemlos. Substantive werden einem grammatischen Geschlecht und einem Flexionstyp zugewiesen, Adjektive und Verben flektieren wie indigene Wörter im unmarkierten Fall. Nehmen wir beispielsweise an, ein Erfolgsautor verweise mit besonderer Sorgfalt auf die Sprache eines Nachrichtenmagazins ${ }^{40}$ und dieses nenne sein Buch im Gegenzug witzig und scharfsinnig, ihn selbst einen Sprachhelden. ${ }^{41}$ Man spricht in einem derartigen Fall treffend von Crosspromotion (ein Femininum). Die substantivische Verlaufsform lautet Crosspromoting (ein Neutrum) und jeder der Beteiligten ist ein Crosspromoter (ein Maskulinum). Die Zuweisung des Genus verläuft reibungslos. Der Plural des Femininums lautet regelgerecht die Crosspromotions und bei lateinischer Leseaussprache (s.u.) die Crosspromotionen. Alles nach den Regeln des Deutschen, nicht des Englischen. Ausnahmen sind jeweils gut begründet und entweder als Übergangstypen anzusehen oder aber als Sonderfälle, die es auch sonst gibt. Strukturelle Auswirkungen des Gebrauchs von Anglizismen sind bisher so gut wie nicht erkennbar. Man findet vermehrt die sog. $s$-Flexion, die durch einen nichtsilbischen Genitiv Singular (des Flops aber des Stuhl(e)s) und einen nichtsilbischen Plural (die Flops aber die Bären) gekennzeichnet ist, wobei der $s$-Plural aber auch unabhängig von den Anglizismen zunimmt. Was die Wortbildung betrifft, ist das Deutsche dabei, mit einigen Wortbausteinen aus dem Englischen produktiv umzugehen, d.h. selbst Wörter zu bilden. Davon zeugen Wortreihen wie Hardcover, Hardliner, Hardrock ... Hardwalk, Hardpop, Hardsound, die unabhängig vom Englischen verlängert werden. Aber auch Affixe wie -ing (Banking) können Analogiebildungen unabhängig vom Englischen und nach Regeln des Deutschen führen. Die dabei entstehenden sog. Pseudoanglizismen gibt es im Englischen nicht, es handelt sich eben um Anglizismen des Deutschen und nicht um Wörter des Englischen. Wir erinnern daran, dass der weitaus größte Teil unserer Latinismen und Gräzismen ebenfalls nicht Entlehnungen, sondern Lehnbildungen sind. Dass man bei Wörtern wie polytoxisch oder operationalisieren nicht von Pseudogräzismen

39 Z.B.: Munske, Horst Haider: Ist das Deutsche eine Mischsprache? Zur Stellung der Fremdwörter im deutschen Sprachsystem. In Munske, Horst Haider u.a. (Hg.): Deutscher Wortschatz. Lexikologische Studien. Berlin 1988, S. 46-74. Eisenberg, Peter: Die grammatische Integration von Fremdwörtern. Was fängt das Deutsche mit seinen Latinismen und Anglizismen an? In: Stickel, Gerhard (Hg.): Neues und Fremdes im deutschen Wortschatz. Aktueller lexikalischer Wandel. Berlin, S. 183-209.

„Wann immer ich an einem Plakat vorbeikam, das auf die ,Kunst des SPIEGEL' hinwies, zischte ich es wie eine Schlange an: ,Sss! Des SPIEGELS!' Da ich bei der Ausstellungseröffnung vermutlich in einen Zisch-Krampf verfallen wäre, bin ich gar nicht erst hingegangen." Sick, Bastian: Der Dativ ist dem Genitiv sein Tod. Folge 3. Köln 2006, S. 73. In der Sache irrt Sick allerdings einmal mehr, denn der Eigenname DER SPIEGEL flektiert anders als die Nominalgruppe der Spiegel mit einem Appellativum als Kern. Die Kunst des SPIEGEL ist eine korrekte Bildung.

41 DER SPIEGEL Nr. 40 vom 2.10.2006, S. 185. 
bzw. Pseudolatinismen spricht, zeigt schon, wie je nach Gebersprache unterschiedlich bewertet wird.

Weniger problemlos verläuft die Integration in der Orthografie und in der Phonetik, wenn auch aus ganz unterschiedlichen Gründen. Lassen Sie mich einige Bemerkungen zur Phonetik machen. Entlehnungen und Lehnbildungen größeren Umfangs führen in der Regel zu Ausspracheproblemen gegenüber dem Kernwortschatz, weil die Lautstruktur der Kernwörter in besonderer Weise restringiert, um nicht zu sagen optimiert ist. Es gehört zu den eindrucksvollsten Erfahrungen des Grammatikers, die Lautkombinatorik und ihre Interaktion mit Wortprosodie und Morphologie zu begreifen. Was die Latinismen und Gräzismen betrifft, hat sich eine recht eindeutige und allgemein akzeptierte Aussprachekonvention (als sog. Leseaussprache) herausgebildet, die in vielerlei Hinsicht mit der des Griechischen und Lateinischen wenig zu tun hat. Für die Gallizismen ist eine solche Konvention weit weniger entwickelt. Einige der Aussprachevarianten von Wörtern wie Chance oder Restaurant, die man im Deutschen zu hören bekommt, tun manchem Sprecher weh. Noch unklarer ist die Lage bei den Anglizismen. Sie ist so unklar, dass es für viele dieser Wörter einfach gar keine Standardaussprache gibt. Der Grund liegt auf der Hand. Anglizismen sind zwar Wörter des Deutschen, behalten aber Eigenschaften des Englischen, einfach weil der Sprachkontakt eng ist. Die im öffentlichen Diskurs vielfach erhobene Forderung nach Integration von Anglizismen geht an der Phonetik vorbei. Gelegentlich wird damit gespielt („,sag doch gleich retsütslen für recyclen"), aber im Allgemeinen umsegelt man das Thema. Besonders widersprüchlich wird es, wenn einerseits geklagt wird, Denglisch sei nicht mal richtiges Englisch, andererseits aber gefordert wird, „Fremdwörter müssen sich grammatisch integrieren lassen. “42

Zweierlei möchte ich aus sprachwissenschaftlicher Sicht hinzufügen. Einen Ausdruck sollte man nicht deshalb stigmatisieren, weil man annimmt, er sei ein Anglizismus. Warum sind Das macht Freude und Das macht Angst gutes Deutsch, nicht aber Das macht Sinn? Und was hat jemand für einen Geschmack, wenn er meint, Das ergibt Sinn sei besseres Deutsch, einmal abgesehen von der Frage, ob beide Ausdrücke tatsächlich dasselbe bedeuten. Warum ist gegen Ausdrücke wie vor 2008, bis 2008, seit 2008 nichts einzuwenden, wohl aber gegen in 2008? Solche Beispiele zeigen, dass wir viele der

42 Zimmer, Dieter E.: Alles eine Frage des Geschmacks? Von wegen! In: DIE ZEIT Nr. 31 2007, S. 43. Bemerkt werden sollte vielleicht, dass die Lautlehre jedenfalls zur Grammatik gehört und dass man die Frage der lautlichen Integration nicht einfach dadurch loswird, dass man dies in Abrede stellt. Sprachkritiker sind häufig gebildete Leute, die ihre Fremdsprachenkenntnisse bei der Aussprache durchaus zur Geltung bringen. S. a. Busse, Ulrich: Probleme der Aussprache englischer Wörter und ihre Behandlung im Anglizismen-Wörterbuch. In: Zettersten, Arne/Pedersen Viggo H. (Hg.): Proceedings of the Seventh International Symposium on Lexicography Copenhagen 1994. Tübingen 1996, S. 83-92. Eisenberg, Peter: Stirbt das Deutsche an den Internationalismen? In: Der Deutschunterricht 51 (1999), H. 2, S. 17-24. 
stigmatisierten Ausdrücke möglicherweise im Deutschen bilden können, ganz unabhängig vom Englischen. Einfach auf die Herkunft hinzuweisen, ist Stammtischgeplauder, das auch zur Stigmatisierung von Wörtern nicht ausreicht.

Schließlich verwahren wir uns als Sprachwissenschaftler gegen einen beliebten Kategorienfehler, der die Sprache selbst an die Stelle des Sprachgebrauchs setzt, meist eines rhetorischen Effekts wegen, z. B.: „Eine starke Beschädigung dieser Folie vermindert die Sprachkompetenz des Einzelnen, und in ihrer Gesamtheit verwüstet sie die deutsche Sprache ... " 43 „Die englischen oder pseudoenglischen Ausdrücke kommen nämlich nicht einfach hinzu ... Sie verdrängen vielmehr die natürliche Wortbildung des Deutschen.“ ${ }^{44}$ „Es geht bergab mit der Sprache, machen wir uns nichts vor. " ${ }^{45}$ "Schreckliches ... Symptom der kranken Sprache aber ist jenes modische Pseudo-Englisch" ${ }^{46}$. „Die Sprache ist in Not.“ ${ }^{47}$ Und vielleicht am gefährlichsten: „Die Lexik einer Sprache ist der (genokulturelle) Code derjenigen ,Kultur, die sich seiner bedient ${ }^{`}$. Zu viele Mutationen auf einmal zerstören den Phänotyp. So entsteht zur Zeit in Deutschland Sprachbruch namens Denglisch ..."48

Schwer vorstellbar, dass jemandem das Deutsche am Herzen liegt, der Derartiges unter seinen Sprechern verbreitet. Mit dem Zustand unserer Sprache hat es nichts zu tun. Die größte Sprache der Europäischen Union ist nicht das Kleinkind, das sich irgendwie von der großen Schwester Englisch abgrenzen müsste.

\section{Wissenschaftssprache: Flexibel und präzise}

Wie eben: Den normalen Sprachteilhaber und zumal einen Wissenschaftler fasst gelegentlich der Zorn, wenn nicht nur an globalistischen BusinessSchools, sondern an ganz normalen Universitäten ganz normale Studiengänge auf das Englische umgestellt werden oder wenn auf wissenschaftlichen Tagungen und in wissenschaftlichen Zeitschriften ein deutsches Publikum auf Englisch angesprochen wird. Ob man, was den Gebrauch als Wissenschaftssprache betrifft, bereits von einem Domänenverlust des Deutschen sprechen muss, hängt davon $a b$, wie man den Begriff Domäne fasst. Unbestreitbar

43 Zimmer, Dieter E.: Deutsch und anders. Die Sprache im Modernisierungsfieber. Hamburg 1997, S. 74.

44 Jessen, Jens: Die verkaufte Sprache. DIE ZEIT Nr. 31 vom 26. Juli 2007, S. 41.

45 Schneider, Wolf: Deutsch! Das Handbuch für attraktive Texte. 2005. Zitiert nach DER SPIEGEL Nr. 40 vom 2.10.2006, S. 183.

46 DER SPIEGEL Nr. 40 vom 2.10. 2006, S. 185

47 DER SPIEGEL Nr. 40 vom 2.10. 2006, S. 198.

48 Dieter, Hermann H.: Does >Denglisch View of a Nature Lover and Language >Fighter . In: Gardt, Andreas/Hüppauf, Bernd (Hrsg.): Globalization and the Future of German. Berlin 2004, S. 139-154. 
bleibt, dass Deutsch in einigen Disziplinen für den internationalen und teilweise den nationalen Gebrauch kaum eine Rolle spielt.

Um Erscheinungen dieser Art begrifflich zugänglich zu machen, ist ein beschreibender Begriff wie Globalisierung von einem ideologischen wie Globalismus zu unterscheiden. ${ }^{49}$ Beschreibt der eine einen historischen Prozess in seinen Facetten, dann erfasst der andere nicht nur unzulässige Abstraktionen und Generalisierungen, sondern noch weiter gehend ein allgemeines, stilles Einverständnis über das, was sowieso passiert. Das kann dazu führen, bestimmte Ereignisse als natürlich oder auch unvermeidlich und berechtigt erscheinen zu lassen, dergestalt, dass die Globalisierung vorangetrieben wird, wo sie es gar nicht müsste und ohne den Globalismus auch nicht könnte. So stellt David Crystal fest, eine Sprache erhalte globale Bedeutung dann, „when it develops a special role that is recognized in every country. " ${ }^{50}$ Nicht das Faktische reicht aus, sondern das allgemeine Einverständnis muss dazukommen. Wir erleben das beispielsweise beim Vormarsch des Englischen im Rahmen des Bologna-Prozesses, der eine sprachliche Harmonisierung eigentlich gar nicht vorsieht.

Was zum Thema Deutsch als Wissenschaftssprache meiner Meinung nach aus sprachwissenschaftlicher Sicht in Kürze zu sagen ist, lässt sich an folgendes Zitat anknüpfen: „,... durch den Verlust der höchsten (internationalen) Redefelder sinkt der Status, das Ansehen der Sprache innerhalb der Sprachgemeinschaft. Eine Schwächung des Status hat immer auch Konsequenzen für den Ausbau der Sprache, für die Arbeit an den Wörtern und Formen, am Korpus der Sprache. “51 Gerade die Formulierung „hat immer auch Konsequenzen" nimmt die sprachwissenschaftliche Autorität des Autors in Anspruch, was ihn vor der Frage des Lesers nach Belegen schützt. Deshalb wird hier einfach festgestellt: Es gibt nicht den leisesten Hinweis auf strukturelle Ausbauhemmungen oder auch nur Ausbauverlangsamungen im Deutschen. Alle Versuche, irgendetwas Konkretes aufzutreiben, sind fehlgeschlagen. Es hat etwas Absurdes, wenn Trabant schreibt „Das Deutsche ... ist damit auf die nationalen Verwendungsweisen reduziert, also auf die Presse, Literatur im engeren Sinne, Verwaltung und Rechtsprechung und nationale Politik " ${ }^{52}$ und

49 Ich stütze mich hier im Wesentlichen auf Haberland, Hartmut: English - The Language of Globalism? Universität Roskilde, Dep. of Culture and Identity (2007). Zu den Wörtern auf -ismus Wellmann, Hans: Zur Problematik einer wissenschaftlichen Sprachpflege: Die „Ismen“. In: Müller, Peter O. (Hg.): Fremdwortbildung. Theorie und Praxis in Geschichte und Gegenwart. Frankfurt/M. 2005, S. 325-343. Eisenberg, Peter: Grundriss der deutschen Grammatik. Band 1. Das Wort. Stuttgart/Weimar 2006 (3. Auf1.). S. $290 \mathrm{ff}$.

50 Crystal, David: The Past, Present, and Future of World English. In: Gardt, Andreas/ Hüppauf, Bernd (Hg.): Globalization and the Future of German. Berlin/New York 2004, S. 27-45, 28.

51 Trabant, Jürgen. In: FAZ vom 28.9.2007, S. 40 (S. Fn. 25).

52 Trabant, Jürgen. In: BBAW, S. 315 (S. Fn. 25). 
diesen Text in einem Band mit ungefähr zwanzig Beiträgen aus unterschiedlichen Disziplinen veröffentlicht, die zeigen, wie flexibel und präzise das Deutsche als Wissenschaftssprache verwendbar ist. Nicht einmal der Hinweis, man werde die Folgen des Domänenverlusts in zwei oder drei Generationen spüren, verfängt. Niemand weiß, welche Rolle das Englische in zwei oder drei Generationen als Wissenschaftssprache spielen wird. Führt man sich die Spekulationen über eine künftige Rolle des Chinesischen vor Augen, ${ }^{53}$ ist gut auch eine neue Rolle für Sprachen wie Französisch oder Deutsch vorstellbar. Zweifel am Ausbau des Deutschen bleiben vorerst unverantwortliches Geplauder, sozusagen der reine Globalismus.

Und was ist gemeint, wenn von den internationalen Redefeldern als den „höchsten" gesprochen wird? Vielleicht muss man nicht unbedingt entscheiden, ob das Globalesische zum heruntergekommenen Englisch oder zum sprachlich Höchsten gehört. Aber irgendwie zusammenbringen muss man die Dinge schon. Ein solcher Versuch findet im öffentlichen Diskurs über den Domänenverlust gar nicht erst statt, auch wenn das Thema in manch anderer Hinsicht differenziert diskutiert wird ${ }^{54}$ Niemand darf ja auch erwarten, das Deutsche als internationale Wissenschaftssprache wäre heute etwa die Sprache von Helmholtz oder Einstein. Viel eher hätte man mit einer Reduktionsform zu rechnen, wie wir sie am Globalesischen besichtigen können. Wären unsere Nostalgiker von einer solchen Sprachausprägung wirklich beglïckt? Egal wie, Hauptsache irgendwie deutsch und irgendwie international?

\section{Sprachscham. Noch heute geschändet?}

Für den Sprachdiskurs wäre einiges gewonnen, wenn wir nicht so täten, als ginge es dem Deutschen besonders schlecht, als hätten wir außergewöhnlich viel Grund zur Klage. Wir fühlen uns, eben wie in deutschen Identitätsdebatten, entweder ganz oben oder ganz verloren. Aber so sehr man das bedauert und so sehr es gelegentlich nervt, so wenig kommt man auch im Sprachdiskurs am deutschen Faschismus vorbei. Er stellt uns in der Tat allein. Der Diskurs über das Deutsche als Sprache des Faschismus hat seinesgleichen nicht. Er beginnt während des „Dritten Reiches“ (natürlich kann man ihn gut auch früher beginnen lassen) und reißt seitdem nicht $a b$. Er hat eine eigene Ge-

53 Instruktiv z. B. die Sonderausgabe „Zeitung der Zukunft“ der Berliner „Tageszeitung“ vom 14. September 2007, dort der Artikel »Leitsprache Chinesisch «. Verschiedene Szenarien werden durchgespielt in Stickel, Gerhard: Unvorgreifliche Erwägungen zum heutigen und zum künftigen Deutsch. In: Liebert, Andreas/Schwinn, Horst (Hg.): Mit Bezug auf Sprache. Tübingen 2009. S. 381-400.

54 Z. B. Pörksen, Uwe (Hg.): Die Wissenschaft spricht Englisch? Versuch einer Standortbestimmung. Göttingen 2005. Ehlich, Konrad: The Future of German and Other Non English Languages of Academic Communication. In: Gardt, Andreas/Hüppauf, Bernd (Hg.): Globalization and the Future of German. Berlin 2004, S. 173-184. 
schichte ${ }^{55}$ und ist durch seine Vielfalt und Intensität prägend für das Sprachbewusstsein eines breiteren Publikums geworden. Zur Vergegenwärtigung nur einige Hinweise.

Unmittelbar nach Ende des Zweiten Weltkriegs beklagt Victor Klemperer, wie wenig sich an der Lingua Tertii Imperii geändert habe. Gerade weil er sich wie kaum ein anderer mit der Wirkung des nationalsozialistischen Sprachgebrauchs befasst hatte, empfindet er die Sprachsituation in Deutschland als bedrückend, ja als bedrohlich. ${ }^{56}$ In dieser Hinsicht durchaus vergleichbar sind die Glossen von Dolf Sternberger, entstanden zwischen 1937 und 1987 und teilweise eingebracht in das „Wörterbuch des Unmenschen “ ${ }^{57}$ Wie bei Klemperer ist an erster Stelle von der Sprache und ihren Wörtern, nicht vom Sprachgebrauch die Rede. Faschismuskritik wird vor allem als Sprachkritik verstanden. In seiner kürzlich erschienenen Auseinandersetzung mit Sternbergers politischer Sprachkritik erinnert William J. Dodd ${ }^{58}$ an den Sprachenstreit der 60er-Jahre, in dem Peter von Polenz und andere Germanisten der jüngeren Generation ihre Wissenschaft gegen eine „panlinguistische Überschätzung der Macht der Sprache über das Denken " ${ }^{59}$ zur Geltung bringen. In der Vorbemerkung zur Ausgabe von 1957 des Wörterbuchs selbst ist bereits von „Sprachgebrauch" die Rede, wo es 1945 einfach „Sprache“ hieß. Das ist für das Folgende von einiger Bedeutung.

In einem noch anderen Sinn politisch lesen wir Adornos Essay über Wörter aus der Fremde. Adorno spricht ebenfalls von der Stellvertreterrolle der Wörter: „Der Sack wird geschlagen, wo der Esel gemeint ist. " ${ }^{60}$ Innerhalb der Germanistik wird daran etwa von Horst Haider Munske mit der Formulierung angeknüpft „Fremdwort trägt die Stigmatisierung bereits in sich ..." ${ }^{61}$ Immer wieder kommt man auf den speziellen Status der Fremdwörter im Deutschen zu sprechen, wird nun direkt oder nur indirekt auf ihre Rolle im

55 Ehlich, Konrad: „..., LTI, LQI, ..." - Von der Unschuld der Sprache und der Schuld der Sprechenden. In: Kämper, Heidrun/Schmidt, Hartmut (Hg.): Das 20. Jahrhundert. Sprachgeschichte - Zeitgeschichte. Berlin 1998, S. 275-303.

56 Klemperer, Victor: Und so ist alles schwankend. Tagebücher Juni bis Dezember 1945. Hg. von Günther Jäckel unter Mitarbeit von Hadwig Klemperer. Berlin 1996. Dazu weiter die Beiträge in Siehr, Karl-Heinz (Hg.): Victor Klemperers Werk. Texte und Materialien für Lehrer. Berlin 2001.

57 Sternberger, Dolf: Aus dem Wörterbuch des Unmenschen und andere Sprachglossen. In: Sternberger, Dolf: Sprache und Politik. Schriften Band IX. Frankfurt a.M./Leipzig 1991, S. 309-426.

58 Dodd, William J.: Jedes Wort wandelt die Welt. Dolf Sternbergers politische Sprachkritik. Göttingen 2007.

59 Polenz, Peter von: Streit über Sprachkritik in den 1960er Jahren. In: Aptum 1 (2005), H. 2, S. 97-111.

60 Adorno, Theodor W.: Wörter aus der Fremde. In: Braun, Peter (Hg.): Fremdwortdiskussion. München 1979, S. 198 (Original 1965).

61 Munske, Horst Haider: Fremdwörter in deutscher Sprachgeschichte. Integration oder Stigmatisierung? In: Stickel, Gerhard (Hg.): Neues und Fremdes im deutschen Wortschatz. Aktueller lexikalischer Wandel. Berlin 2001, S. 7-29. 
Nationalsozialismus verwiesen. Besondere Verdienste um die Erforschung des Wortgebrauchs im Nachkriegsdeutschland hat die Düsseldorfer Schule erworben $^{62}$ und erwirbt sie weiter. Denn inzwischen geht es um die Bewertung von belasteten Wörtern in der zweiten und dritten Generation. Was sagt man da$\mathrm{zu}$, wenn ein Vierzigjähriger äußert, man werde im kommenden Jahr „die Endlösung " für den Fahrplan des Nahverkehrs in einer deutschen Großstadt finden? ${ }^{63}$

Die sprachwissenschaftliche Auseinandersetzung mit dem deutschen Faschismus hat zahlreiche weitere Facetten, von Arbeiten zur Sprache von Emigranten deutscher Zunge nach Israel ${ }^{64}$ über die Einrichtung eines Fachs Jiddistik ${ }^{65}$ bis zur Erforschung des Sprachgebrauchs im Deutschland der ersten Nachkriegszeit. ${ }^{66}$

Dieser Teil des öffentlichen Diskurses hat, das wurde ja schon deutlich, für manchen Sprachwissenschaftler meiner Generation eine durchaus biografische, persönliche Seite. Es geht um das Reden von der „Sprache des Nationalsozialismus", mit dem wir aufgewachsen sind. Erfahrungen meiner Generation etwa bei Auslandsaufenthalten in den späten 50er- und frühen 60erJahren haben die Redeweise zunächst verfestigt. Es gab tatsächlich eine Sprachscham, die vielleicht sogar dazu beitrug, dass wir mit besonderem Eifer Französisch oder Englisch lernen wollten.

Ein Umdenken setzte mit der Sprachkritik der 60er-Jahre und einer gründlichen politischen Auseinandersetzung mit dem Nationalsozialismus in den späten 60er-Jahren ein, wie sie beispielsweise in der Zeitschrift „Das Argument" stattfand. Ich will gar nicht versuchen, den Gesamtkontext dieses Vorgangs und seine zeitgeschichtliche Bedeutung zu illustrieren oder gar zu erfassen, schon gar nicht im Jahr der 68er-Jubiläumspeinlichkeiten. Die Sprache des Nationalsozialismus kam den jungen Sprachwissenschaftlern näher und schließlich so nahe, dass sie verschwand. Die Nationalsozialisten hatten nicht die deutsche Sprache zugrunde gerichtet, sondern hatten sie in einer Weise verwendet, in der man auch andere Sprachen vor ihr und mit ihr

62 Stötzel, Georg/Eitz, Thorsten (Hg.): Zeitgeschichtliches Wörterbuch der deutschen Gegenwartssprache. Schüsselwörter und Orientierungsvokabeln. Hildesheim 2003 (2. Auf1.). Wengeler, Martin (Hrsg.): Sprachgeschichte als Zeitgeschichte. Hildesheim 2005.

63 Eitz, Thomas/Stötzel, Georg: Wörterbuch der ,Vergangenheitsbewältigung“. Die NSVergangenheit im öffentlichen Sprachgebrauch. Hildesheim 2007. Dazu auch Dieckmann, Walther: „Belastete Wörter" als Gegenstand und Resultat sprachkritischer Reflexion. Aptum 3 (2007), H. 1, S. 62-80. Unterstöger, Hermann: Der heikle Sprachalltag. In: Süddeutsche Zeitung vom 11. 3. 2008, S. 22.

64 Betten, Anne/Du-nour, Miryam: Wir sind die Letzten. Fragt uns aus. Gespräche mit den Emigranten der dreißiger Jahre in Israel. Gerlingen 1995 und Gießen 2004.

65 Lehrveranstaltungen zur Jiddistik gibt es z. B. an den Universitäten Düsseldorf und Trier.

66 Zeitreflexion im ersten Nachkriegsjahrzehnt 1945-1955. Forschungsprojekt am IDS Mannheim (Leitung Heidrun Kämper). Das Projekt ist abgeschlossen, eine Monografie in Vorbereitung. 
verwendet hat. Am Wissen von unserer Alleinstellung änderte das nichts, es konnte dann jedoch eine Arbeit unseres Kollegen Gerhard Voigt mit dem Titel Bericht vom Ende der "Sprache des Nationalsozialismus" ${ }^{67}$ erscheinen. Sie war alles andere als Ausdruck eines „Jetzt reichts“ oder „Einmal muss Schluss sein“. Die jüngere Generation von Sprachwissenschaftlern hat sich der Arbeit am Deutschen in dem Bewusstsein verschrieben, dass diese Sprache schwer missbraucht worden war. Die Scham ist geblieben. Es ist die Scham über den Faschismus, nicht über die deutsche Sprache.

Aber auch das Gerede von der perversen und geschändeten Sprache geht weiter. So gehört es nach wie vor zur amerikanischen Gruselkultur, den Charakter des Deutschen als natürliche Sprache unter den anderen natürlichen Sprachen ruhig einmal infrage zu stellen: „Kann es eine Sprache geben, die so pervers, so verdreht, so sadistisch ist, dass sie ihren Sprechern in der Mehrheit der Fälle irreguläre Formen aufzwingt? " 68 Noch bedauerlicher ist, wenn der Diskurs über das Deutsche an der Sprachscham der gesamten Sprachgemeinschaft aufgehängt wird und dies auch noch mit sprachwissenschaftlicher Autorität: „Ich glaube nun, dass diese Erinnerung, das Gefühl der Schuld und der Scham, eine spezifisch deutsche ,Sprachscham', die sprachhistorischen Veränderungen, die ähnlich derzeit auch in anderen europäischen Sprachgemeinschaften stattfinden, stark beeinflusst. Die deutsche Sprachscham gibt diesen sprachhistorischen Entwicklungen hierzulande ihre ganz besondere Dramatik. " 69

Damit wären wir zurückgefallen auf die Sprache selbst, so als hätte niemand etwas dazugelernt und als hätte sich seit den 60er-Jahren nichts verändert. Das eigene Sprachtrauma („Ich leide an ihr und liebe sie doch“) wird, gnadenlos verallgemeinert, zum Angriff auf die Sprache. Der Angriff hat seine Tücken, weil er politisch korrekt, politisch opportun und im Ornat fachlicher Fundiertheit daherkommt. Die Fakten trifft er nicht, einer Sorge um die Sprache kann er nicht geschuldet sein. Entfaltet er Wirkung, wird er ihr Schaden zufügen. Das unaufhörlich flotte Geplauder sollte sich einer simplen sprachwissenschaftlichen Aufklärung anbequemen, an der es ja keineswegs fehlt. ${ }^{70}$ Und erneut lernen wir: Die Sprachwissenschaft hat sich dem öffentlichen Diskurs zu stellen, weil er zeigt, wo der größte Bedarf an Aufklärung besteht. Damit wird sie der Verantwortung für ihren wunderbaren Gegenstand am ehesten gerecht.

67 Voigt, Gerhard: Bericht vom Ende der „Sprache des Nationalsozialismus“. In: Diskussion Deutsch 19 (1974), S. 445-469.

68 Pinker, Steven: Wörter und Regeln. Die Natur der Sprache. Aus dem Englischen übersetzt von Martina Wiese. Heidelberg/Berlin 2000, S. 255.

69 Trabant, Jürgen: BBAW, S. 311 f. (S. Fn. 25). Die BBAW stellt Trabants Artikel in die Rubrik „Akademische Causerie“, zu Deutsch „Akademische Plauderei“. Daran tut sie gut.

70 Feilke, Helmut/Knobloch, Clemens/Völzing, Paul-Ludwig (Hg.): Was heißt linguistische Aufklärung? Sprachauffassungen zwischen Systemvertrauen und Benutzerfürsorge. Heidelberg 2007. 